\title{
Sorafenib-induced acute-on-chronic liver failure in a patient with hepatocellular carcinoma after transarterial chemoembolization and radiofrequency ablation: A case report
}

\author{
QING-LIANG WANG ${ }^{1 *}$, XIAO-JIE LI ${ }^{2 *}$, ZHI-CHENG YAO ${ }^{1}$, \\ PENG ZHANG $^{1}$, SHI-LEI XU ${ }^{1}$, HE HUANG ${ }^{1}$ and KUN-PENG HU ${ }^{1}$ \\ Departments of ${ }^{1}$ General Surgery and ${ }^{2}$ Laboratory Medicine, Lingnan Hospital, The Third Affiliated \\ Hospital of Sun Yat-sen University, Guangzhou, Guangdong 510530, P.R. China
}

Received April 6, 2016; Accepted September 14, 2016

DOI: $10.3892 / \mathrm{mco} .2017 .1363$

\begin{abstract}
Comprehensive treatments together with sorafenib provide a survival benefit for patients with advanced hepatocellular carcinoma (HCC). Acute-on-chronic liver failure (ACLF) is an increasingly recognized distinct entity; however, only few cases induced by sorafenib have been reported to date. We herein present a rare case of ACLF in a patient under treatment with sorafenib. A 63-year-old woman who suffered from hepatitis B for 10 years was admitted to our hospital. A computed tomography (CT) scan led to a diagnosis of HCC in the V/VIII hepatic segment, with invasion of the middle hepatic and the right portal veins. The patient received multidisciplinary treatment, including transarterial chemoembolization, radiofrequency ablation and sorafenib. Two months after sorafenib treatment, the patient was readmitted due to progressive jaundice and ACLF was diagnosed by liver biopsy shortly thereafter. Although aggressive treatment was administered, the patient succumbed to the disease following rapid deterioration of her clinical condition. The majority of patients with HCC have underlying liver disease and combination therapy with sorafenib should be administered with caution, as it may increase the risk of severe hepatotoxicity. The present case suggests that patients treated with sorafenib may require a dose reduction following interventional treatment.
\end{abstract}

Correspondence to: Dr Kun-Peng Hu, Department of General Surgery, Lingnan Hospital, The Third Affiliated Hospital of Sun Yat-sen University, 2693 Kaichuang Avenue, Luogang, Guangzhou, Guangdong 510530, P.R. China

E-mail: hkpdhy918@126.com

${ }^{*}$ Contributed equally

Key words: acute-on-chronic liver failure, sorafenib, hepatocellular carcinoma, transarterial chemoembolization, radiofrequency ablation

\section{Introduction}

Hepatocellular carcinoma (HCC) is a leading cause of cancer-related mortality worldwide and its incidence is increasing (1). The majority of patients with HCC are unsuitable for curative surgical resection. However, multimodal approaches, such as transarterial chemoembolization (TACE) and radiofrequency ablation (RFA), may be used in clinical practice. Although there are no widely accepted diagnostic criteria, acute-on-chronic liver failure (ACLF) is a newly recognized clinical entity characterized by sudden deterioration of a chronic underlying liver disease (2). Sorafenib is a multikinase inhibitor that targets both Raf and vascular endothelial growth factor and platelet-derived growth factor receptor tyrosine kinase signaling. More recently, sorafenib has come to be considered as the standard treatment for patients with advanced HCC. Liver dysfunction was reported in $<1 \%$ of sorafenib-treated patients in the SHARP and Asia-Pacific trials $(3,4)$ and only few cases of severe sorafenib-induced hepatitis have been described. We herein present the case of a patient who developed ACLF with a fatal outcome during treatment with sorafenib following TACE and RFA.

\section{Case report}

A 63-year-old woman was admitted to Lingnan Hospital (Guangzhou, China) in September, 2013 due to upper abdominal discomfort, fatigue, anorexia and a weight loss of $2 \mathrm{~kg}$ within 1 month. The patient has suffered from hepatitis B for 10 years without any medical treatment. Other past medical history and family history were unremarkable. Upon admission the patient was asymptomatic and there was no evidence of hepatic encephalopathy, ascites or peripheral edema. Physical examination revealed mild abdominal distention without tenderness, while the findings of the gynecological examination were normal.

The laboratory results revealed microcytic anemia (haemoglobin $9.3 \mathrm{~g} / \mathrm{dl}$, haematocrit $28 \%$, mean corpuscular volume $63 \mathrm{fl})$. Liver tests revealed mild hypoalbuminemia $(3.3 \mathrm{~g} / \mathrm{dl}$; normal, 3.5-5.5 g/dl), aspartate aminotransferase (AST) $51 \mathrm{U} / 1$ (normal range, 10-40 U/l) and alanine aminotransferase (ALT) 

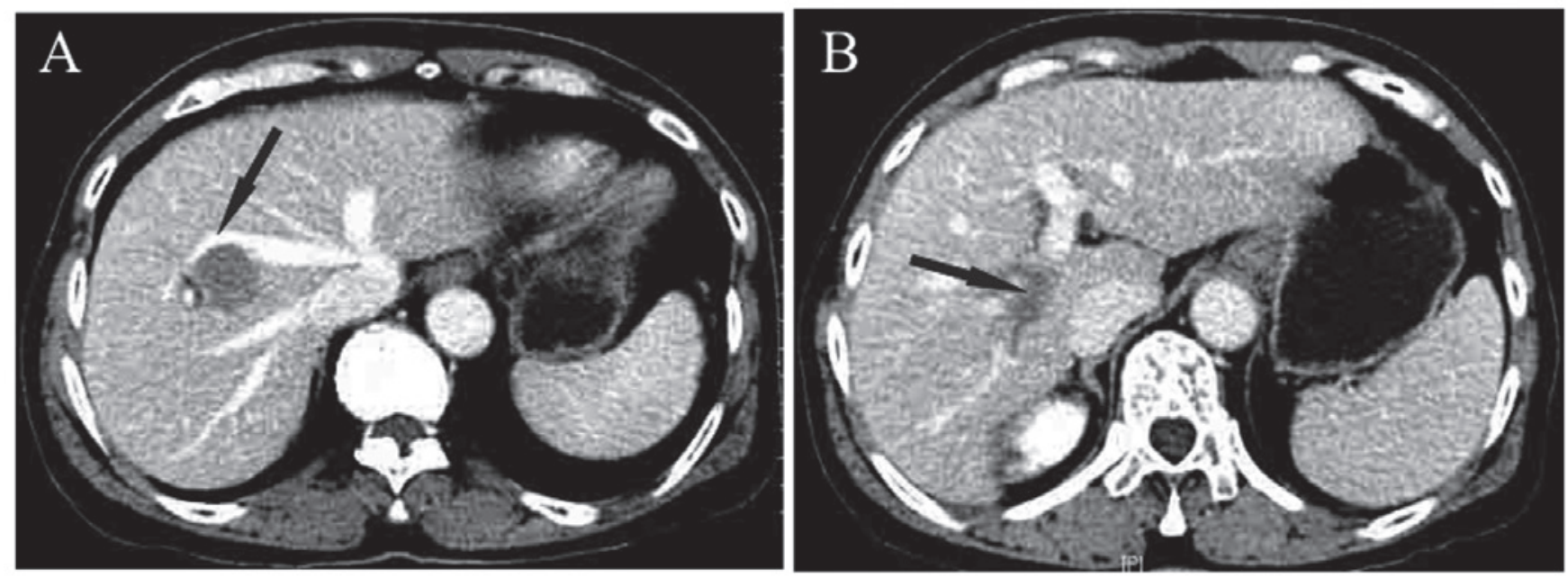

Figure 1. Abdominal computed tomography scan showing a unifocal tumor in the V/VIII hepatic segment with invasion of (A) the middle hepatic vein and (B) the right portal vein (arrows).

$68 \mathrm{U} / 1$ (normal range, 7-56 U/l), whereas all other liver function tests were normal. The serum tumor markers $\alpha$-fetoprotein (AFP), carbohydrate antigen (CA)19-9, CA-125, CA-153 and carcinoembryonic antigen (CEA) were all within the normal range. The following hepatitis $B$ virus (HBV) markers were also detected: $\mathrm{HBsAg}^{+} ; \mathrm{HBeAg}^{-} ; \mathrm{HBeAb}^{+} ; \mathrm{HBcAb}^{+}$; and HBV-DNA 2.84x 107 copies/ml. Abdominal contrast-enhanced computed tomography $(\mathrm{CT})$ revealed a $3.0 \times 2.6-\mathrm{cm}$ unifocal tumor in the V/VIII hepatic segment (Fig. 1).

As the liver lesion invaded the middle hepatic and right portal veins, the patient was unsuitable for potentially curative surgical resection. TACE was used to treat the primary cancer and, at the same time, comprehensive treatments, such as effective inhibition of HBV replication, were applied. The patient successfully recovered and was discharged 10 days after TACE. Following diagnosis with advanced incurable HCC, sorafenib was initiated the first week of October at a dosage of $400 \mathrm{mg}$ twice daily. The side effects of sorafenib were generally mild, including anorexia, tinnitus, nausea and vomiting, which were well-controlled medically. One month later, a repeated CT scan of the liver indicated that the initial tumor had not completely regressed following treatment. In November, ultrasound-guided RFA was performed. The patient recovered soon and sorafenib treatment, which interrupted immediately before and after RFA, was resumed.

Seven weeks later, the patient was readmitted with abdominal pain, progressive jaundice and abdominal distension. The liver function parameters were as follows: ALT $970 \mathrm{U} / 1$, AST $1166 \mathrm{U} / 1$, total bilirubin/conjugated bilirubin $335 / 204 \mu \mathrm{mol} / 1$, albumin $26 \mathrm{~g} / 1$, prothrombin time $39 \mathrm{sec}$ and activated partial thromboplastin time $54 \mathrm{sec}$. HBV DNA was repeatedly undetectable and AFP remained within the normal range. These results were consistent with the diagnosed characteristics of ACLF. Liver biopsy revealed intrahepatic cholestasis, parenchymal necrosis, inflammatory infiltrates, as well as significant fibrosis, indicating both acute and chronic liver injury (Fig. 2). Although several therapies were applied, the patient succumbed to severe liver decompensation after 10 days. Written informed consent was obtained from the

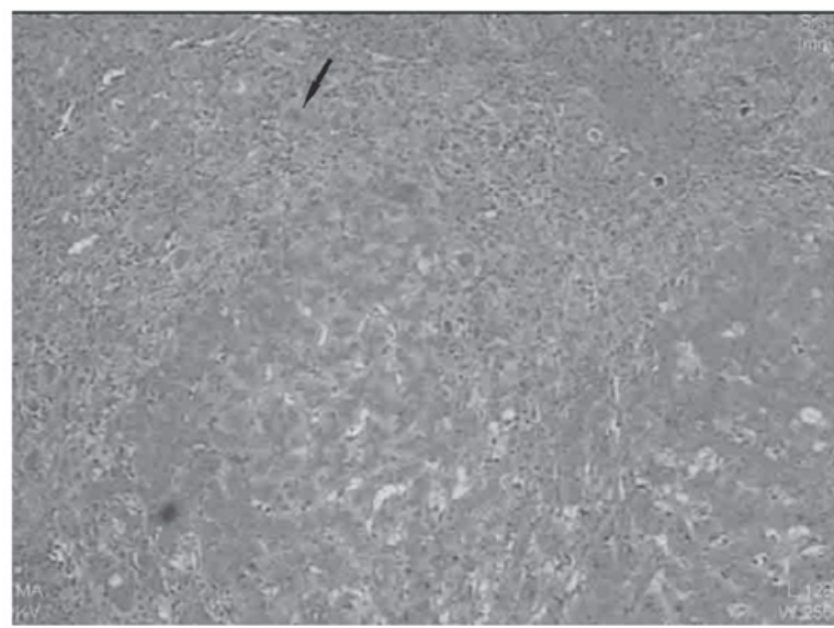

Figure 2. Pathological result of the biopsy showing intrahepatic cholestasis (arrow), inflammatory infiltrates and fibrosis. Hematoxylin and eosin staining; magnification, x100.

patient's family for the publication of the case details and associated images.

\section{Discussion}

There are multiple treatment options for HCC, including liver resection, local ablation, TACE or selective internal radiotherapy, targeted therapy with sorafenib and liver transplantation (5). The majority of the cases with HCC are diagnosed at an advanced stage and, therefore, patients cannot benefit from curative therapy. For our patient, as the primary liver tumor invading the major veins, a multidisciplinary treatment approach was applied.

TACE has become the first choice of treatment for patients with non-surgical HCC. However, the outcome in the present case was not satisfactory due to the aggressive nature of the tumor. The SHARP and Asia-Pacific trials demonstrated a significant survival benefit and good tolerance in patients with 
advanced HCC, making sorafenib the new reference standard for systemic therapy of patients with advanced $\operatorname{HCC}(3,4)$. Ineffective TACE is associated with a negative harm-benefit balance, without prolongation of the overall survival, and should not be repeated. TACE failure is an indication for sorafenib therapy in patients with advanced HCC (6). Based on these analyses, sorafenib was administered at a dose of $400 \mathrm{mg} / \mathrm{bid}$. TACE and the specific anti-angiogenesis effect of sorafenib is one of the major strategies for a combination treatment approach. The CT scan after TACE indicated that the initial tumor did not fully regress. RFA is considered a safe and feasible procedure, with a low complication rate; thus, ultrasound-guided RFA was also applied to control tumor progression.

Hyperbilirubinemia is almost invariably present and jaundice is considered an essential criterion for the diagnosis of ACLF. In addition to jaundice, another hallmark of liver dysfunction is coagulopathy. Prolongation of the prothrombin time is common. Coagulation tests are usually abnormal in cirrhotic patients due to impaired synthesis and increased consumption of coagulation factors. In the present case, the liver function parameters revealed that the total bilirubin was $335 \mu \mathrm{mol} / 1$ and the prothrombin time was $39 \mathrm{sec}$. The clinical symptoms after hospitalization also include the development of hepatic encephalopathy, ascites and hepatorenal syndrome.

To date, the most commonly reported sorafenib-related adverse events have been diarrhea, fatigue, rash, alopecia, anorexia and nausea. The side effects in our patient were mild and effectively controlled. Liver failure was uncommon among sorafenib-treated patients in the SHARP and Asia-Pacific trials $(3,4)$. However, Ozenne et al reported that $7(21 \%)$ French patients with Child-Pugh A class liver disease experienced liver failure (7). In a retrospective study from Japan, Ogasawara et al also reported a higher frequency of liver failure (8): Of the 54 patients treated, liver failure occurred in $10(19 \%)$.

ACLF is a rare syndrome with diverse etiology, which is associated with a high mortality rate (9). Although the exact pathogenesis remains to be elucidated, a number of causes may contribute to the development of ACLF. In Asia, the continuous replication and expression of $\mathrm{HBV}$ is considered as one of the most common acute inducers leading to ACLF (10). Another acute precipitant for ACLF is infection. Infection plays an important role in the progression and management decisions of ACLF. However, there was no evidence of HBV reactivation or specific infection after hospitalization.

The therapeutic challenge in this patient was that treatment had to be performed in a patient with an already damaged cirrhotic liver, which by itself causes significant morbidity and mortality and increases drug toxicity. Recent studies also indicated that the effectiveness of the combination of sorafenib and TACE remains questionable (11) and two recent meta-analyses reported discrepant results $(12,13)$. However, the combination of TACE and sorafenib induced two-way targeting of vascularization through embolization and inhibition of angiogenesis. Thus, we considered that, as Ogasawara et al had reported, patients treated with sorafenib may require a dose reduction.

In conclusion, although rare, the possible development of ACLF in patients undergoing sorafenib treatment must be kept in mind. Our experience highlights that combination therapy with sorafenib should be applied with caution and patients treated with sorafenib may require a dose reduction following interventional treatment.

\section{Acknowledgements}

The present study was supported by a grant from the Science and Technology Program of Guangdong Province (no. 2014A020212715).

\section{References}

1. Gomaa AI and Waked I: Recent advances in multidisciplinary management of hepatocellular carcinoma. World J Hepatol 7: 673-687, 2015.

2. Jalan R, Gines P, Olson JC, Mookerjee RP, Moreau R, Garcia-Tsao G, Arroyo V and Kamath PS: Acute-on chronic liver failure. J Hepatol 57: 1336-1348, 2012.

3. Llovet JM, Ricci S, Mazzaferro V, Hilgard P, Gane E, Blanc JF, de Oliveira AC, Santoro A, Raoul JL, Forner A, et al: Sorafenib in advanced hepatocellular carcinoma. N Engl J Med 359: 378-390, 2008

4. Cheng AL, Kang YK, Chen Z, Tsao CJ, Qin S, Kim JS, Luo R, Feng J, Ye S, Yang TS, et al: Efficacy and safety of sorafenib in patients in the Asia-Pacific region with advanced hepatocellular carcinoma: A phase III randomised, double-blind, placebo-controlled trial. Lancet Oncol 10: 25-34, 2009.

5. Bruix J and Sherman M; American Association for the Study of Liver Diseases: Management of hepatocellular carcinoma: An update. Hepatology 53: 1020-1022, 2011.

6. Arizumi T, Ueshima K, Chishina H, Kono M, Takita M, Kitai S, Inoue T, Yada N, Hagiwara S, Minami Y, et al: Validation of the criteria of transcatheter arterial chemoembolization failure or refractoriness in patients with advanced hepatocellular carcinoma proposed by the LCSGJ. Oncology (87 Suppl 1): 32-36, 2014.

7. Ozenne V, Paradis V, Pernot S, Castelnau C, Vullierme MP, Bouattour M, Valla D, Farges O and Degos F: Tolerance and outcome of patients with unresectable hepatocellular carcinoma treated with sorafenib. Eur J Gastroenterol Hepatol 22: 1106-1110, 2010.

8. Ogasawara S, Kanai F, Obi S, Sato S, Yamaguchi T, Azemoto R, Mizumoto H, Koushima Y, Morimoto N, Hirata N, et al: Safety and tolerance of sorafenib in Japanese patients with advanced hepatocellular carcinoma. Hepatol Int 5: 850-856, 2011.

9. Jalan R and Williams R: Acute-on-chronic liver failure: Pathophysiological basis of therapeutic options. Blood Purif 20: 252-261, 2002.

10. Liu Q, Liu Z, Wang T, Wang Q, Shi X and Dao W: Characteristics of acute and sub-acute liver failure in China: Nomination, classification and interval. J Gastroenterol Hepatol 22: 2101-2106, 2007.

11. Kudo M, Imanaka K, Chida N, Nakachi K, Tak WY, Takayama T, Yoon JH, Hori T, Kumada H, Hayashi N, et al: Phase III study of sorafenib after transarterial chemoembolisation in Japanese and Korean patients with unresectable hepatocellular carcinoma. Eur J Cancer 47: 2117-2127, 2011.

12. Fu QH, Zhang Q, Bai XL, Hu QD, Su W, Chen YW, Su RG and Liang TB: Sorafenib enhances effects of transarterial chemoembolization for hepatocellular carcinoma: A systematic review and meta-analysis. J Cancer Res Clin Oncol 140: 1429-1440, 2014.

13. Liu L, Chen H, Wang M, Zhao Y, Cai G, Qi X and Han G: Combination therapy of sorafenib and TACE for unresectable HCC: A systematic review and meta-analysis. PLoS One 9: e91124, 2014. 